\title{
Coherent transition radiation from attosecond electron pulses
}

\author{
Maxim Tsarev ${ }^{1, *}$ and Peter Baum ${ }^{1,2,3, *}$ \\ ${ }^{1}$ Ludwig-Maximilians-Universität München, Am Coulombwall 1, 85748 Garching, Germany \\ ${ }^{2}$ Max-Planck-Institute of Quantum Optics, Hans-Kopfermann-Str. 1, 85748 Garching, Germany \\ ${ }^{3}$ Universität Konstanz, Universitätsstraße 10, 78464 Konstanz
}

\begin{abstract}
We show theoretically and by simulations how coherent transition radiation from tilted surfaces can be used for characterization of attosecond free-electron pulses such as used for pump-probe electron microscopy and diffraction. The tilted geometries eliminate velocitymismatch and beam-diameter effects, providing sensitivity to attosecond times even for almost arbitrarily large beam diameters.
\end{abstract}

\section{Introduction}

Attosecond electron pulses produced by optical modulation allow pump-probe diffraction and microscopy of sub-light-cycle dynamics [1,2]. Here we study whether coherent transition radiation (CTR) is a viable method for electron pulse characterization. Transition radiation is generated whenever a charged particle passes a boundary between two media. In case of short pulses, the emitted radiation carries information about the pulse shape in space and time. In principle, the emitted bandwidth is inversely proportional to the pulse duration, but in typical geometries the emitted spectrum is substantially damped in the short-wavelength region by the influence of the lateral size of the electron beam [3], which causes destructive optical interference that prevents the direct characterization of attosecond pulses.

Here we introduce two different tilted mirror geometries that solve the problem by providing velocity matching [4]. Basically, we disentangle the spectrum from the spatial contribution and thus achieve attosecond resolution over almost arbitrarily large beam diameters. We compute the overall efficiency for realistic beams and find that attosecond pulse metrology by transition radiation is indeed a feasible concept for extreme electron pulse characterization.

\section{Tilted geometries for velocity-matched emission of coherent transition radiation}

The spectral-angular distribution of CTR energy emitted by a bunch consisting of $N$ electrons is given by [5]

\footnotetext{
*Corresponding authors: maxim.tsarev@1mu.de, peter.baum@uni-konstanz.de
} 


$$
W_{\Omega, \omega} \approx W_{\Omega, \omega}^{1} N^{2}|\tilde{f}|^{2}
$$

where $W_{\Omega, \omega}^{1}$ is the incoherent emission spectrum of a single particle and $\tilde{f}$ is the normalized-to-unity 3D Fourier-transform of the charge density in the bunch. In contrast to incoherent transition radiation of a single particle, CTR is a second order process $\left(\sim N^{2}\right)$ and thus allows reconstruction of the bunch's temporal profile from the emitted spectra.

If an electron beam (blue) with the velocity $v$ hits a foil or surface (black) at normal incidence (Fig. 1a), a short pulse of finite beam diameter impinges everywhere at the same time and each part (each single electron) emits coherently with a single-cycle behaviour in time, that is, with a broadband spectrum. However, at an off-axis detector, which is required because there is no emission in the backwards direction [5], the individual singleelectron emissions interfere destructively as a consequence of the finite beam diameter.

(a)

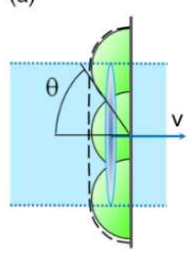

(b)

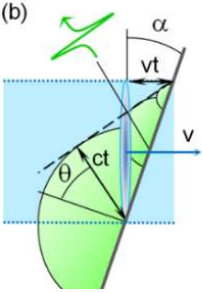

(c)

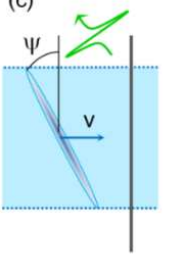

(d)

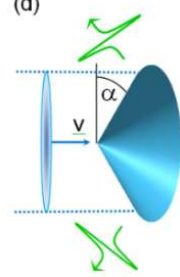

Fig. 1. Concept of using coherent transition radiation (CTR) for detection of ultrashort electron bunches. (a) For normal incidence, no emission is observed because of destructive interference . (b) For a properly tilted surface, velocity matching provides in-plane constructive interference for all frequencies emitted to a certain angle independently of the lateral size of the bunch. (c) A properly tilted electron pulse provides a similar effect. (d) A conically-shaped surface in a cylindrical geometry fully eliminates the beam's transverse-size influences on the CTR in any plane of emission .

However, if the surface is tilted by a properly chosen angle $\alpha$ (Fig. 1b), all individual dipole emissions (single-cycle in time and broadband) arrive coherently at a given detector angle $\theta$ with constructive interference and in temporal synchrony [4]. This velocitymatching condition can be written down as

$$
v \sin \theta=c \sin \alpha .
$$

Mathematically, the transverse-size dependency of the bunch's form-factor $\tilde{f}$ is eliminated at $\theta$ given by Eq. (2) and simultaneously $\tilde{f}$ is maximized. Practically, the detector angle $\theta$ can be chosen to maximize single-particle emission $W_{\Omega, \omega}^{1}$, and the tilt angle chosen according to Eq. (2). The highest emitted frequency is now independent of the beam diameter and solely depends on the electron pulse duration in the propagation direction. For a Gaussian pulse with a $1 / \mathrm{e}$ duration $\tau$, the peak emitted wavelength is $\lambda_{\text {peak }}=\sqrt{2} \pi c \tau$. Visible and ultraviolet radiation can therefore be expected for attosecond electron pulses. An even more efficient geometry is a conically-shaped surface (Fig. 1d), which provides velocity matching for any emission plane. The expected conical emission contains the best possible total energy, if a cylindrically-shaped detector is applied.

\section{Results and discussion}

Figure 2 shows the calculated spectral and angular distributions 3-fs, 1-fs and 100-as for a nearly ideal mirror as the radiator. We see that, indeed, ultraviolet radiation is expected for attosecond electron pulses, characterizing their duration. For estimations of the 
transition radiation for a realistic mirror, we numerically analysed a set of available materials as potential CTR radiators [4]. We find that metallic interfaces, such as silver, are well-suited for the electron pulses of $\sim 0.5$ fs and longer. For shorter pulses, we find that silicon is a good material for pulses down to 100 as. We estimated the overall yield of CTR for a number of experimental situations. For example, pulse trains of 480 -as electron pulses from Ref. [1] in a multi-electron regime (3 electrons per pulse) and in the geometry of Fig. $1 \mathrm{~d}$ with a silver radiator would produce about $\sim 6000$ photons per second in the wavelength range from 0.3 to $3 \mathrm{~mm}$. This flux is realistically measurable and provides a simple way for attosecond electron pulse metrology, probably easier than an optical oscilloscope [6] or waveform electron microscopy [7]. The concept should also allow to tackle the quantumoptical properties of delocalized attosecond single-electrons within a pulse train $[1,2]$.

(a)

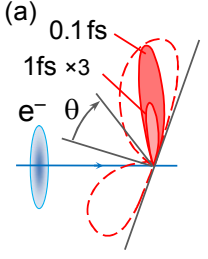

(b)

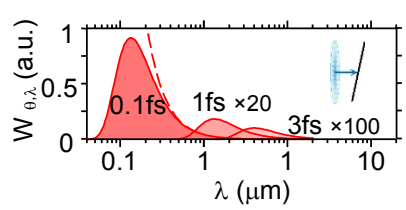

(c)

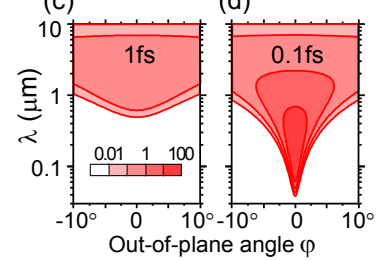

Fig. 2. Frequency-angular spectra of CTR for $\tau=3 \mathrm{fs}, 1 \mathrm{fs}$ and $0.1 \mathrm{fs}$ (shaded areas) and corresponding single-particle spectra (dashed curves) in the properly tilted geometry $\left(\alpha=18^{\circ}\right.$, optimizes emission at $\theta=68^{\circ}$ ). Bunch transverse radius (1/e) is $3 \mu \mathrm{m}$. (a) Radiation patterns at $\lambda=1 \mu \mathrm{m}$. (b) Spectra at $\theta=68^{\circ}$. (c) Out-of-plane emission distribution at $\theta=68^{\circ}, \tau=1 \mathrm{fs}$. (d) The same but for $\tau=100$ as.

To conclude, tilted emitter geometries provide velocity-matching and eliminate the transverse-size dependency of coherent transition radiation spectra. Due to the direct relation between emission wavelength and pulse duration, our concept allows to measure pulse durations down to 100 attoseconds in a direct and passive way. The concept should also be useful for characterizing few-attosecond pulses, should they eventually be generated.

The authors acknowledge funding by the European Research Council (project DIVI) and the Munich Centre for Advanced Photonics. This project has received funding from the European Union's Horizon 2020 research and innovation programme under Marie Sklodowska-Curie grant agreement No 713694 .

\section{References}

1. Y. Morimoto, P. Baum, Nature Phys. 14, 2526 (2018)

2. K. E. Priebe, C. Rathje, S. V. Yalunin, T. Hohage, A. Feist, S. Schäfer, C. Ropers, Nature Photon. 11, 793 (2017)

3. C. J. Hirschmugl, M. Sagurton, G. P. Williams, Phys. Rev. A 44, 1316 (1991)

4. M. V. Tsarev, P. Baum, New J. Phys. 20, 033002 (2018).

5. V. L. Ginzbung, V. N. Tsytovich, Transition Radiation and Transition Scattering (CRC Press, New York, 1990)

6. C. Kealhofer, W. Schneider, D. Ehberger, A. Ryabov, F. Krausz, P. Baum, Science 352, 429 (2016)

7. A. Ryabov, P. Baum, Science 353, 374 (2016) 